\title{
Can Mathematics be Biology's next microscope in disease research at the interface?
}

\section{Keynote presentation at the 50th anniversary conference of the Mammal Research Institute of University of Pretoria}

\author{
Roumen Anguelov *, Armanda Bastos ${ }^{\dagger}$ \\ * Department of Mathematics and Applied Mathematics \\ $\dagger$ Mammal Research Institute, Department of Zoology and Entomology \\ University of Pretoria, South Africa \\ roumen.anguelov@up.ac.za, adbastos@zoology.up.ac.za
}

\begin{abstract}
In this paper we discuss how mathematics can be integrated into biological research, as well as the benefits and challenges related to this process. The focus is on research of diseases at the interface between wildlife, humans and livestock with some illustrative examples of the applications of mathematical models to disease research that we have personally been involved with.
\end{abstract}

Keywords-mathematical modelling; thresholding; sensitivity analysis; endemicity; extinction

\section{INTRODUCTION}

Mathematical models and methods have become important tools for research in the biosciences in general, and for population dynamics and disease epidemiology in particular [4]. In this paper we focus primarily on research at the disease interface, however many of the issues dealt with are of more general relevance.

Disease research at the interface (henceforth denoted "the interface") refers to the study of infectious disease transmission at the interface between free-ranging wildlife on the one side and livestock and / or humans on the other. Demographic and socio-economic pressures force wildlife, humans and their domestic animals to co-exist in close proximity, thus intensifying disease transmission potential at the interface. The interface could be linear (e.g. along a fence), or patchy, reflecting habitat preference of disease hosts. It could be focal (at a point), or diffuse, when a range of resources are shared over wide area [2]. Wherever the interface occurs, disease transmission is potentially bi-directional. Indigenous diseases that are typically maintained in wildlife may cross the interface to livestock or humans (eg. foot and mouth disease, African swine fever, ebola), whereas diseases that are exotic to an area are often introduced by human activity and may cross the interface to wildlife (eg. bovine tuberculosis, canine distemper, rinderpest). A specific challenge for research at the interface arises from the two highly divergent approaches to managing humantransformed landscapes versus natural areas within which wildlife are generally confined. Whilst strict veterinary and health policies are applied in the 
former, a policy of least human intervention is applied to the later. This increased complexity as well as specific factors such as limited opportunities for observation, difficulties and restrictions in collecting data, cost of conducting experiments (if at all possible), etc., make the use of mathematical models and methods a relevant and appropriate research approach.

Mathematical modelling can be considered as the process of embedding existing knowledge into a mathematical construct. The embedded knowledge is of (i) a qualitative nature, e.g. the law of mass action for the interaction of two species, or of (ii) a quantitative nature, e.g. the space density of each species at given times. Further, and possibly equally important, one can embed in the model hypotheses in order to test their feasibility and validity. The need for mathematical modelling is motivated by the substantially high complexity of the biological phenomena considered by contemporary biosciences. Indeed, due to technological advances, the possibilities of studying an entire system rather than individual elements or aspects as well as collecting measurements of many observable variables at the same time has increased tremendously. Further, solutions to problems of conservation, agriculture, health are sought in the context of a larger system and the long term sustainability analysis is founded on a good understanding of the dynamics of this system. Mathematics provides a suitable medium, where the existing qualitative and quantitative knowledge of the system can be adequately represented as a model. The theoretical analysis and practical simulation may produce results which are beyond the reach of normal observation, experimentation or intuition, thus, giving substance to the statement that "Mathematics is Biology's next microscope" [4].

\section{INTEGRATING MATHEMATICS INTO BIOLOGICAL RESEARCH}

The classical modus operandi of using mathematical/statistical methods in biological research follows the sequence: Research question biological observations and/or experiments - collection of data - mathematical/statistical analysis. This typical modus operandi, reflects the limited use of mathematical methods, which are often only restricted to the analysis of the data, e.g. establishing correlations ${ }^{1}$ Such approaches seldom result in a practically relevant and useful model. We would like to suggest that for mathematical modelling to be efficient and useful it needs to be considered an integral part of all stages of the respective research project. For example, the research question can be considered in the setting of a mathematical model which represents the existing knowledge, allowing for identification of gaps, the relative importance of the involved parameters, the dependence of the parameters of interests on the observable variable as well as any pre-existing bifurcation states. The experimental and field work can be informed by these finding so that when data are collected, hypotheses within the model can be accepted or rejected, aspects of the model can be improved, and the values of parameters of interest reliably identified. The mathematical analysis can also reveal the need for further research leading to a more complete and realistic model, which is also useful for providing answers to the original research questions, possibly generating additional questions along the way. A forum for bringing mathematical expertise and/or collaboration at the early stages of a project is essential for the proposed integration of mathematics into biological research and should form a central tenet upon which future research initiatives are planned.

\section{OUTPUT OF MATHEMATICAL MODELS AND THEIR ANALYSIS}

In order to avoid being too general, and so that practically meaningful conclusions can be reached, we will mainly consider models representing dynamical systems, typically formulated in terms of ordinary and/or partial differential equations. Possible outputs of the analysis of these models are listed below.

\footnotetext{
${ }^{1}$ Correlation should not be confused with causality as the former does not imply the latter
} 
- Internal consistency of the set of assumption and/or established knowledge represented via the equations of the model.

- Bifurcation (thresholding) analysis providing the parameter domains of qualitatively different behaviour of the system and characterising this behaviour, e.g. species invading or not, pathogen persisting or becoming extinct.

- Testing the validity of assumptions (hypotheses) by comparing model properties with existing observations of the system.

- Identify the values of parameters of interest by using the values of known parameters and data on the values of observable variables (microscope function).

- Identify knowledge gaps.

- Project scenarios into the future (timetelescope function).

- Quantify uncertainty (guaranteed range, confidence intervals, sensitivity).

To illustrate these benefits we provide examples from our own work on mathematical modelling of two infectious vector-borne disease agents: Bartonella and African swine fever virus (ASFV). The Barnonella model [3], considers two vertebrate hosts, Rattus rattus and Rattus norvegicus and two vectors, ticks (Ixodidae family) and fleas (genus Xenopsylla). The model takes into account the infection dynamics in each of the four species interlinked via host to vector and vector to host forces of infection (Figure 1). The model itself is written in terms of ordinary differential equations. It provides interesting insights on the infection dynamics and the relative importance of its parameters. In particular, the high sensitivity of the model on the rate of vertical transmission motivated further research, conducted as part of an MSc study which aimed to accurately estimate its value. Further, the mathematical analysis proved that differences in the ectoparasite load between the two Rattus species cannot alone explain the difference in their Bartonella infection rates.

The main aim of the development of the ASFV model was to investigate a possible natural mechanism for a locally occurring extinction and was

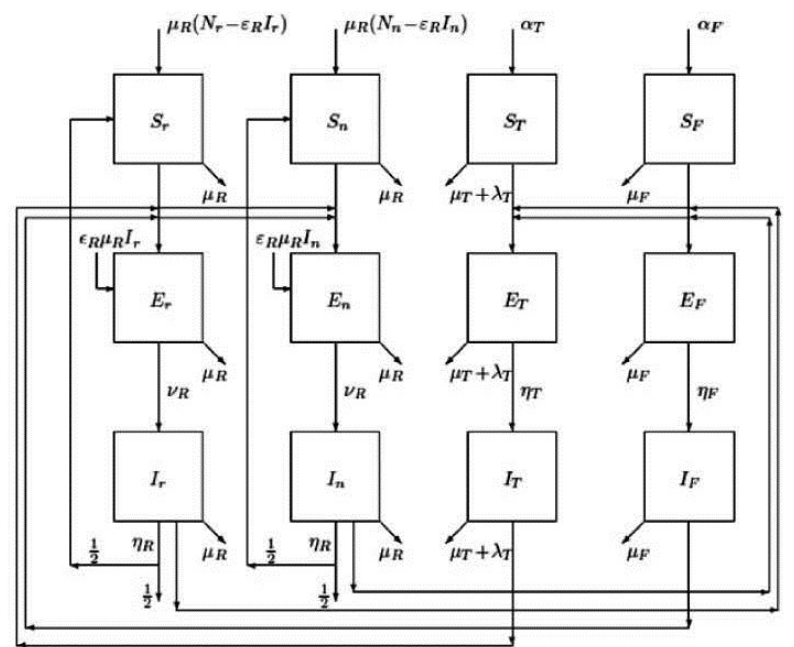

Fig. 1. Bartonella in $R$. rattus, $R$. norvegicus, ticks and fleas (S-susceptible, E-exposed, I-infective, R-recovered).

motivated by the apparent disappearance of the virus from a previously positive tick population in the Mkuze Game Reserve, South Africa [1]. The model focuses on the infection dynamics within a burrow infested with Ornithodoros soft ticks. The movements of warthog sounders in or out of the burrow are relatively fast processes and thus modeled via impulses. The presence of naïve warthogs in the burrow is an important seasonal virus amplification event for the tick population. The flow chart represented in Figure 2 is implemented in terms of a system of impulsive differential equations (ordinary and partial) [8].

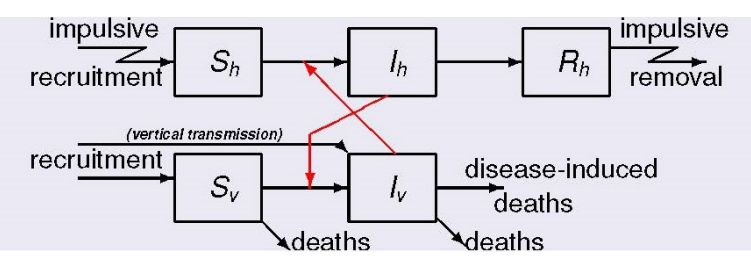

Fig. 2. ASVF in warthogs (vertebrate host) and ticks (vector) in a burrow, impulsive recruitment $=$ warthog sounder moving in (S-susceptible, E-exposed, I-infective, R-recovered).

The model reveals that the prevalence in a burrow is maintained via the interplay between the mentioned virus amplification event and vertical transmission in ticks. Disrupting this mechanism 
(burrow not inhabited by neonatal warthogs for sufficiently long time) may lead to virus extinction.

\section{Challenges to Mathematics}

Despite the abstract nature of mathematics, its development is primarily driven by human practicalities. Physics was for a long time the main inspiration and driver behind many mathematical theories. In contemporary research, Biology is increasingly assuming its place [4] and poses new challenges for mathematics. Here we will list two that are particularly relevant to dynamical systems.

- Analysis of nonlinear dynamical systems. Biological systems are highly nonlinear. Despite all of the achievements, the theories of Analysis and the Analysis of Differential Equations are largely linear theories. Examples that can be included: (i) Linear diffusion vs Nonlinear diffusion (ii) Nonlinear death rate and extinction in finite time.

- Dealing with uncertainty. Parameters of models of biological systems are typically only known to within a particular range rather than to have a precise value. Mathematical theories dealing with set-valued data need further development. One approach is via Interval Analysis, [7].

Constructing mathematical models also poses challenges for biology:

- Quantitative analysis of interactions. Mathematical models are built on knowledge of the causal relationships between the model variables, e.g. populations, subpopulations, factors, etc., resulting in adequate functional representation of these relationships, e.g. the number of new infections is proportional to the number of existing infectives. Such knowledge is seldom available even on topics that have been well researched. If biologists and epidemiologists are serious about using mathematical modelling, future research needs to give attention to the quantitative analysis of functional links between the intended model variables.
- Relative completeness of knowledge. A mathematical model is a complete construct in the sense that it functions autonomously with prescribed data and parameters. Therefore a model can be useful only if based on adequate knowledge of all the main contributing factors to the studied phenomenon. When constructing a model we necessarily make simplifying assumptions. We take into account some factors, e.g. the life cycle of a disease vector and disregard others (e.g. the phase of the moon when a vector bites the host mammal), based on their (possibly perceived) impact. The model needs knowledge on all relevant aspects of all factors considered of importance.

\section{INTEGRATED APPROACH TO DISEASE STUDIES AT THE INTERFACE}

The processes at the interface arise from interactions between transformed habitats (inhabited by humans and domestic/ated animals) on the one side and natural ecosystems (with wildlife) on the other, in turn result from processes inherent to each of these two sides as well as to global abiotic factors such as climate. Integrating knowledge on these three components can potentially provide better insight into disease dynamics and opportunities for control. The usefulness of some links have been recognised, e.g. seasonal calving of wildebeest and the risk of malignant catarrhal fever for cattle [9], or earth satellite images used in identifying vegetation as a proxy for predicting the distribution of dung beetles and herbivores [6]. The opportunities for such integrated approaches have increased with the availability of computer technology for storing vast amounts of data as well as algorithms to search and link data from different sources and in different formats the so called Big Data science. In this setting an integrated approach has the potential to be an important factor driving future mammal research and research on diseases at the interface. In particular, it facilitates mathematical modelling since a mathematical model can embed relevant knowledge from all disciplines and spheres of research. 


\section{MAmmal Research AT University of PRETORIA}

The University of Pretoria's Mammal Research Institute (MRI) celebrated its 50th anniversary by convening a four-day conference from 12th to 16th September 2016 at Mopani Camp in Kruger Park. The conference was aimed at setting the stage for the next 20 years of African mammal research activities in the face of ongoing socio-political, socio-economic and environmental changes and drafting a blueprint to guide ongoing and new initiatives that will prepare graduates, scientists, policy makers and NGOs to cope with these anticipated challenges. These initiatives were centered around three main themes:

(i) People and wildlife;

(ii) Environmental stressors;

(iii) Diseases at the interface between wildlife, livestock and humans.

The keynote talk presented in this paper relates to the theme "Diseases at the Interface".

The field of Biomathematics has been actively promoted over the last decade and was given a formal status in South Africa by the establishment in 2013 of the DST/NRF SARChI Chair on Mathematical Methods and Models in Biosciences and Bioengineering. The Department of Mathematics and Applied Mathematics is a partner to the MRI and to other institutes and centers of biological research. The integrative approach promoted in this paper has resulted in a number of initiatives such as an informal weekly meeting under the name of "Biomath Coffee" which facilitates the early involvement of mathematics in biological research. Within this forum presentation of student projects still at the stage of formulation of research question are encouraged, with presenters benefitting from the comments and advice of biologists and mathematicians present and, at least in some cases, fruitful collaboration was established [3], [5], [6].

As previously mentioned, an integrated approach to mammal research and specifically to disease studies at the interface may benefit substantially by using Big Data Science. In this regard, the recent establishment of an institute for Big Data at the University of Pretoria is a timely initiative.

\section{CONCLUSION}

This paper considers some essential principles in relating mathematics to biological research, in general, and to epidemiological research on diseases at the interface between wildlife, humans and livestock, in particular. The impact of the interaction between mathematics and biosciences on each of these two disciplines as well as the benefits of this interaction for each discipline are discussed with comment on recent developments and initiatives that will facilitate this further. The exposition is not meant to be a complete text on the topic. It has the much more modest aim of raising issues of importance and giving some pointers to guide integrative, inter-disciplinary research.

\section{REFERENCES}

[1] L.F. Arnot, J.T. Du Toit,A.D.S. Bastos, Molecular monitoring of African swine fever virus using surveys targeted at adult Ornithodoros ticks: a re-evaluation of Mkuze Game Reserve, South Africa, Onderstepoort Journal of Veterinary Research 76(2009), 385-392.

[2] R.G. Bengis, R.A. Kock, J. Fisher, Infectious animal diseases: the wildlife/livestock interface, Scientific and Technical Review of the Office International des Epizooties 21(1)(2002), 53-65

[3] H. Brettschneider, R. Anguelov, Chimimba, A.D.S. Bastos, A mathematical epidemiological model of gramnegative Bartonella bacteria: does differential ectoparasite load fully explain the differences in infection prevalence of Rattus rattus and Rattus norvegicus?, Journal of Biological Dynamics 6(2)(2012), 763-781

[4] J.E. Cohen, Mathematics Is Biology's Next Microscope, Only Better; Biology Is Mathematics' Next Physics, Only Better. PLoS Biol 2(12): e439 (2004), doi:10.1371/journal.pbio.0020439

[5] C. Dufourd, C. Weldon, R. Anguelov, Y. Dumont, Parameter Identification in Population Models for Insects Using Trap Data , Biomath 2 (2013), 1312061, http://dx.doi.org/10.11145/j.biomath.2013.12.061

[6] I. Engelbrecht, M. Robertson, M. Stoltz, J.W. Joubert, Reconsidering environmental diversity (ED) as a biodiversity surrogacy strategy. Biological Conservation 197(2016), 171179

[7] S. Markov, Biomathematics and Interval Analysis: A Prosperous Marriage, AIP Conf. Proc. 1301(26) (2010), http://dx.doi.org/10.1063/1.3526621 
R Anguelov, A Bastos, Can Mathematics be Biology's next microscope in disease research at the interface?

[8] P. Sivakumaran, Mathematical epidemiological models with finite time extinction: the case of African swine fever virus, MSc thesis, University of Pretoria, 2016.

[9] L. Wambua, P.N. Wambua, A.M. Ramogo, D. Mijele, M.Y. Otiende, Wildebeest-associated malignant catarrhal fever: perspectives for integrated control of a lymphoproliferative disease of cattle in sub-Saharan Africa, Archives of Virology 161(2016), 1-10 